\title{
Sex-related Alterations of Gut Microbiota in the C57BL/6 Mouse Model of Inflammatory Bowel Disease
}

\author{
Hee Jin Son ${ }^{1}$, Nayoung Kim ${ }^{1,2}$, Chin-Hee Song ${ }^{1}$, Ryoung Hee Nam ${ }^{1}$, Soo In Choi ${ }^{1}$, Joo Sung Kim ${ }^{2}$, Dong Ho Lee ${ }^{1,2}$ \\ ${ }^{1}$ Department of Internal Medicine, Seoul National University Bundang Hospital, Seongnam, ${ }^{2}$ Department of Internal Medicine and Liver Research \\ institute, Seoul National University College of Medicine, Seoul, Korea
}

\begin{abstract}
Background: Gut microbiota is closely associated with development and exacerbation of inflammatory bowel diseases (IBD). The aim of this study was to investigate differences in gut microbiota depending on sex and changes of gut microbiota during IBD developments. Methods: 16s rRNA metagenomic sequencing was performed for fecal materials from 8-week-old wild type (WT) and interleukin 10 (IL-10) knockout (KO) C57BL/6 mice of both sexes. Diversity indices, relative abundance of microbiota, and linear discriminant analysis effect size were examined to compare microbial communities between groups. Clustering of groups was performed by principal coordinates analysis (PCOA) and unweighted pair group method with arithmetic mean (UPGMA). Functional capabilities of microbiota were estimated using phylogenetic investigation of communities by reconstruction of unobserved states (PICRUSt) based on Kyoto Encyclopedia of Genes and Genomes database.

Results: PCOA and UPGMA tree analysis of beta-diversity demonstrated significant differences in gut microbiota between male and female groups of WT mice, but not of IL-10 KO mice. Firmicutes to Bacteroides ratio was higher in male group than that in female group in both WT mice and IL-10 KO mice. Phylum Proteobacteria significantly increased in female IL-10 KO mice than that in female WT mice. At species level, Lactobacillus murinus, Bacteroides acidifaciens, and Helicobacter hepaticus significantly increased in IL-10 KO mice than in WT mice. The relative abundance of beta-glucuronidase (K01195) was higher in female IL-10 KO mice than that in female WT mice by PICRUSt.

Conclusions: Our results suggest that microbiota-host interactions might differ between sexes during development of IBD.

(J Cancer Prev 2019;24:173-182)
\end{abstract}

Key Words: Gut microbiota, Sex differences, Inflammatory bowel disease, Sequence analysis, Interleukin-10

\section{INTRODUCTION}

Inflammatory bowel disease (IBD) including Crohn's disease (CD) and ulcerative colitis is a chronic inflammatory disorder of gastrointestinal tract with a broad range of clinical manifestations [1]. A number of susceptibility genes have been discovered, suggesting the importance of genetic predisposition in IBD [2-4]. Recently, interruption of dynamic balance between gut microbiota and human host has been highlighted as a possible pathogenesis of IBD in a genetically susceptible individual [5]. In healthy individuals, intestinal immune tolerance allows coexistence of resident gut microbiota with hosts. However, hosts with susceptible conditions have modified compositions of gut microbiota and altered host immune regulations which might eventually lead to disease development [6]. An absence of intestinal inflammation in interleukin 10 (IL-10) knockout (KO) mice, an animal model of IBD, without gut bacteria supports the importance of gut microbiota in IBD pathogenesis [7]. Clinical trials of IBD treatments trying to adjust dysbiosis have also shown positive results [8,9]. A randomized controlled trial on effects of antibiotics in IBD treatment has shown that rifamycin can significantly improve induction of remission in CD [8]. Fecal microbiota transplantation has also gained attention as a potential treatment strategy for IBD by resolving dysbiosis [9].

Received September 1, 2019, Revised September 25, 2019, Accepted September 25, 2019

Correspondence to: Nayoung Kim

E-mail: nayoungkim49@empas.com, ORCID: Nayoung Kim, https://orcid.org/0000-0002-9397-0406

Copyright (C) 2019 Korean Society of Cancer Prevention

(c) This is an Open Access article distributed under the terms of the Creative Commons Attribution Non-Commercial License (http://creativecommons.org/licenses/by-nc/4.0) which permits unrestricted non-commercial use, distribution, and reproduction in any medium, provided the original work is properly cited. 
Following the progression of metagenomics and next generation sequencing, understanding of gut microbiota has been deepened. While characteristics of individual bacterial species have been focused in past studies, characteristics of bacterial species as communities are being focused on recently. Various studies have reported differences in relative abundance and diversities of gut microbiota between healthy and IBD groups in animal models and humans [10-13]. However, these studies have not shown consistent results due to their difference in study conditions, such as diverse animal models, sample sites, sampling methods, storage conditions, and sexes [10-15]. Moreover, whether the relationship between gut microbiota and the disease is a causation or a correlation has not been determined yet [16].

To more thoroughly understand interactions between microbiota and hosts related to intestinal inflammation and IBD, numerous possible pathways regarding immunologic, metabolic, and hormonal perspectives have been considered [17]. Short-chain fatty acids (SCFAs) are metabolites derived from microbiota that have been focused. SCFAs can induce modulations of immune responses by differentiating immune cells or promoting immune cytokine secretion [18,19]. Lack of proper immune development in germ-free animals emphasizes critical roles of gut microbiota in host immune system development [20,21]. Estrobolome, a group of microbial products related to estrogen metabolism, is a recently arising concept that suggests sex hormones like estrogen could contribute to explain how microbiota and host interact $[17,22]$.

From this background, we hypothesized that sex hormones could interact with gut microbiota and acquire different microbial communities that might finally result in different IBD features between sexes. Thus, the aim of this study was to investigate sex differences of gut microbiota in $\mathrm{C} 57 \mathrm{BL} / 6$ mice regarding microbial diversities, relative abundance, and functional capabilities. In addition, we tried to evaluate how sex differences might alter as intestinal inflammation progressed in IL-10 KO mice, an IBD animal model. This concept is consistent with epidemiologic data showing sex differences in incidence of IBD [23,24].

\section{MATERIALS AND METHODS}

\section{Animals and fecal sample collection}

Male and female specific pathogen-free (SPF) wild type (WT) mice and IL-10 KO C57 mice with age of eight weeks obtained from Orient Bio Inc. (Seongnam, Korea) were used in this study. Experiments were conducted with four groups: male WT group, female WT group, male IL-10 KO group, and female IL-10 KO group ( $\mathrm{n}=8$ mice per group). Mice were housed in a humiditycontrolled room at $23^{\circ} \mathrm{C}$ with an alternating 12-hour light/dark cycle under SPF condition. They were provided with unrestricted access to food and water. Feces were collected immediately after defecation. All fecal samples were immediately frozen in liquid nitrogen and stored at $8^{\circ} \mathrm{C}$.

All protocols used in this study were approved by the Institutional Animal Care and Use Committee (IACUC) of Seoul National University Bundang Hospital (permission number: BA1506-178/039-01). All procedures were conducted in accordance with the Guide for the Care and Use of Laboratory Animals of South Korea.

\section{Metagenome sequencing of $16 \mathrm{~S}$ rRNA gene}

Total DNAs were extracted from fecal samples using QIAamp ${ }^{\circledR}$ DNA stool mini 245 kits (Qiagen, Venlo, Netherlands) following the manufacturer's recommendations. To prepare MiSeq library amplicons, target gene (V3-V4 region of 16S rRNA gene) was amplified using 341F (5'-TCGTCGGCAGCGTCAGATGTGTATAAG AGACAG CCTACGGGNGGCWGCAG-3') and 805R (5'-GTCTCGTG GGCTCGG AGATGTGTATAAGAGACAGGACTACHVGGGTATCTAA TCC-3') primers. PCR products were confirmed by electrophoresis and purified using a QIAquick PCR 250 purification kit (Qiagen). V3-V4 PCR amplicons were attached with Illumina indices and adapters from Nextera ${ }^{\circledR}$ XT Index Kit (Illumina, San Diego, CA, USA). Short DNA fragments were eliminated using a FavorPrep ${ }^{\text {TM }}$ DNA purification kit (Favorgen, Pintung, Taiwan). PCR amplicons were quantified with a Quant-iTTM PicoGreen ${ }^{\mathrm{TM}}$ dsDNA Assay Kit (Thermo Fisher Scientific, Waltham, MA, USA). After pooling samples (300 ng per sample) PCR products were purified with a FavorPrep ${ }^{\mathrm{TM}}$ DNA gel extraction kit (Favorgen).

Quality assessment for confirmation of the DNA integrity and product size was conducted on a Bioanalyzer 2100 instrument (Agilent, Santa Clara, CA, USA) using a DNA 7500 chip at ChunLab, Inc. (Seoul, Korea). Metagenome sequencing was performed using Illumina MiSeq platform at ChunLab, Inc.

Raw reads were processed starting with quality check and filtering of low quality $(<$ Q25) reads using Trimmomatic 0.32 [25]. Paired-end sequence data were merged using PANDAseq [26]. Primer sequences were then trimmed with an in-house program of ChunLab, Inc. at a similarity cut off of 0.8 . Nonspecific amplicons that did not encode $16 \mathrm{~S}$ rRNA were identified by using HMMER program hmmsearch with 16S rRNA profiles [27]. Sequences were denoised using DUDE-Seq [28]. Nonredundant reads were extracted through UCLUST clustering [29]. EzBioCloud database was utilized for taxonomic assignment with USEARCH 
(8.1.1861_i86linux32) [29] followed by more precise pairwise alignment [30]. UCHIME [31] and nonchimeric 16S rRNA database from EzBioCloud were used to detect chimeras for reads that had best hit similarity rate of less than $97 \%$. Sequence data were then clustered using CD-HIT [32] and UCLUST [29].

\section{Metagenome sequencing data analysis}

Microbial diversity (observed operational taxonomic unit [OTU] count, ACE, Chaol, Jackknife, Shannon, Simpson indices, and Phylogenic diversity, Good's library coverage) was examined using BIOiPLUG (Chunlab, Inc.). To visualize sample differences, principal coordinates analysis ( $\mathrm{PCOA}$ ) was performed with a generalized UniFrac method. Clustering of samples was explained with principal coordinate values. An unweighted pair group method with arithmetic mean (UPGMA) tree was created using BIOiPLUG (ChunLab, Inc.) [33]. Taxonomic summary bar charts were created for OTU abundance ratio (\%) at phylum and family level using GraphPad Prism (ver. 8.01).
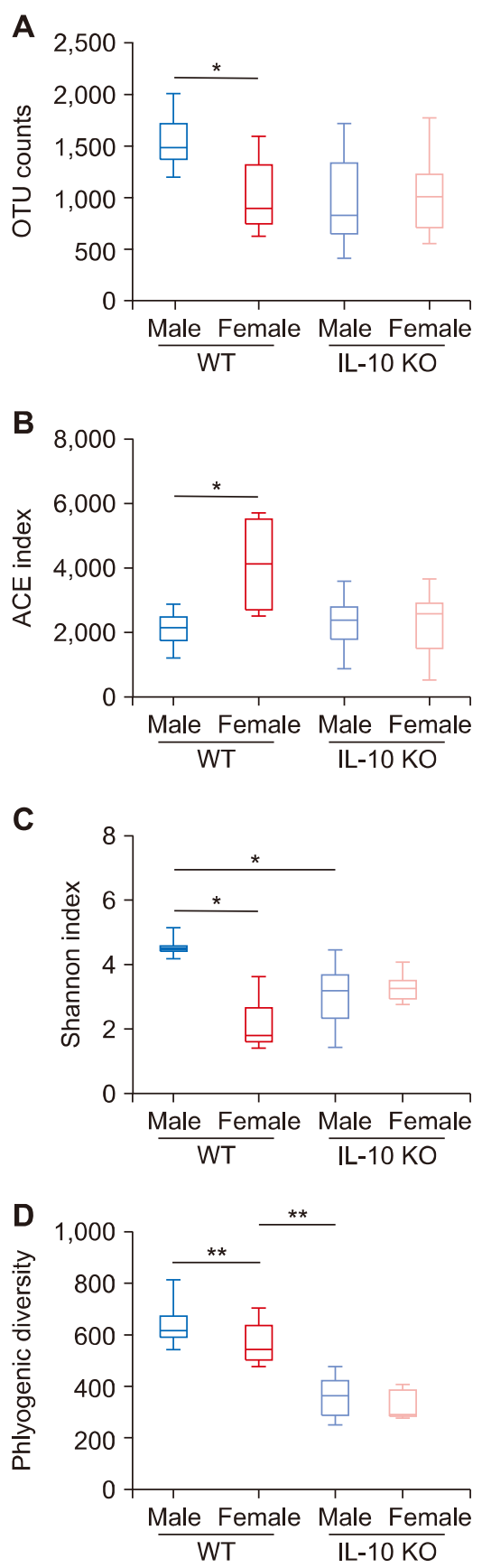

E

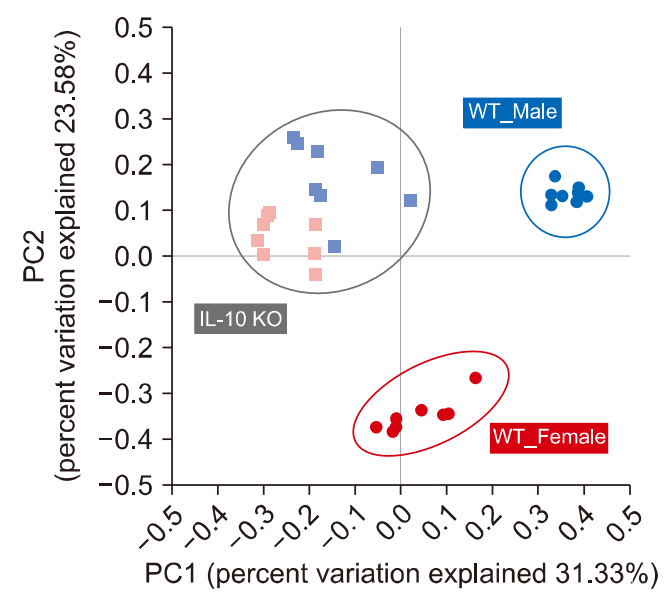

$\mathbf{F}$

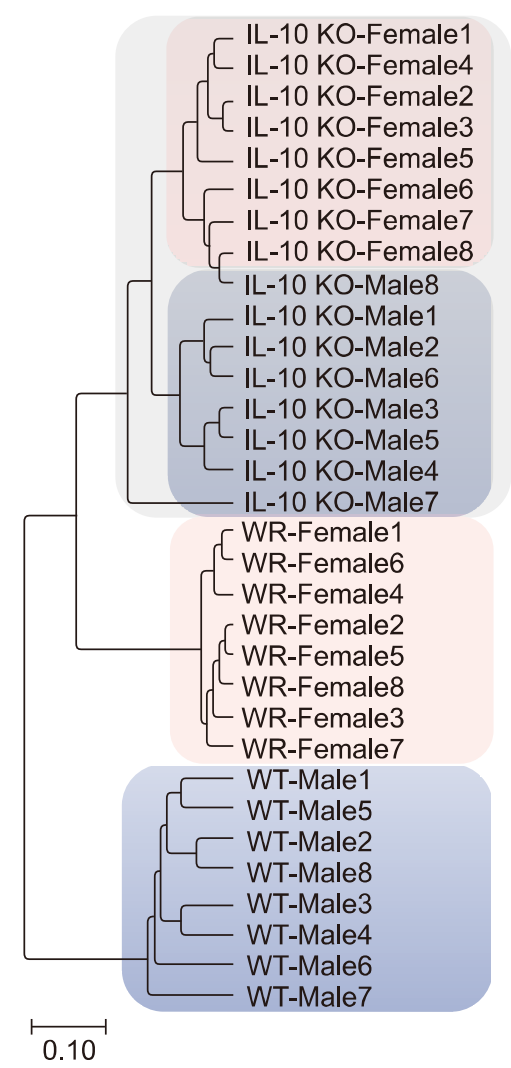

Figure 1. Diversity indices of gut microbiota. Microbiota richness: (A) operational taxonomic unit [OTU] counts and (B) Accumulated Cyclone Energy [ACE]; and diversity: (C) Shannon (D) phylogenic diversity) in wild type (WT) mice and interleukin 10 (IL-10) knockout (KO) mice of both sexes. (A-D) $P$-value of Kruskal-Wallis $<0.05$ for. Beta diversity analyses of Principal coordinates plot (E) and Unweighted Pair Group Method with Arithmetic mean tree demonstrate that samples are clustered into distinct groups by sex and genotypes $(\mathrm{F}) .{ }^{*} P<0.013$. ** $P<0.0025$ after Holm-Bonferroni correction. 
Linear discriminant analysis (LDA) effect size (LEfSe) analysis was conducted in order to determine taxonomic compositions that were significantly changed regarding sex and IL-10 KO [34]. Conditions of LEfSe analysis were: (1) an alpha value for factorial Kruskal-Wallis test between control and high fat diet (HFD) of less than 0.05; (2) an alpha value for pairwise Wilcoxon test among taxonomic compositions of less than 0.05; (3) a threshold of logarithmic LDA score for discriminative features of less than 2.0; and (4) multi-class analysis set as all-against-all.

To predict functional capabilities of microbial community, we performed phylogenetic investigation of communities by reconstruction of unobserved states (PICRUSt) [35]. This analysis enabled us to infer alterations in functional markers of microbiota based on Kyoto Encyclopedia of Genes and Genomes database.

\section{Statistical analysis}

Statistical calculations other than those for pyrosequencing data were performed using PASW Statistics ver. 18.0, 2009 (IBM Corp., Armonk, NY, USA). Groups were compared through Kruskal-Wallis test, followed by Mann-Whitney U-test with Holm-Bonferroni correction. Results with $P$-value less than 0.05 were considered statistically significant.

\section{Accession number}

The raw unprocessed 16S rRNA gene sequence, which was generated by our study was deposited in the National Center for Biotechnology Information Sequence Read Archive Database (SRA accession number PRJNA564389).

\section{RESULTS}

\section{Alterations of microbiota diversities related to sex and interleukin 10 knockout}

Male and female WT mice showed significant sex differences in species richness and diversity indices of gut microbiol communities (Fig. 1, Table 1). Specifically, ACE index estimated higher species richness in female WT mice compared to male WT mice (Fig. 1B), while diversity indices Shannon and Simpson's indicated that male WT mice had higher microbial diversity than female WT mice (Fig. 1C, Table 1). Male and female IL-10 KO mice showed significant decrement of phylogenic diversity compared to their WT counterparts (Fig. 1D). Male IL-10 KO mice also had smaller Shannon index value than male WT group (Fig. 1C).

PCoA analysis and UPGMA tree demonstrated that WT groups and IL-10 KO groups were significantly classified into two different groups. Moreover, male WT mice and female WT mice were significantly separated into two distinct groups (Fig. 1E and $1 \mathrm{~F})$. The difference between male IL-10 KO mice and female IL-10 KO mice was less marked than that between male WT mice and female WT mice.

\section{Effect of sex differences on relative abundance of gut microbiota in C57BL/6 mice}

Male and female WT mice showed different compositions of gut microbiota at both phylum and family taxa levels. Phyla Bacteroidetes and Firmicutes were almost equally dominant in male WT mice, while phylum Bacteroidetes was the most prominent one among others in female WT mice (Fig. 2A, Fig. 3B and $3 \mathrm{C}$ ). Firmicutes to Bacteroidetes ratios ( $\mathrm{F} / \mathrm{B}$ ratios) were smaller in female groups than those in their male counterparts of both WT and IL-10 KO mice (Fig. 3A).

Table 1. Basic characteristics and diversity indices of gut microbiota

\begin{tabular}{|c|c|c|c|c|}
\hline \multirow{2}{*}{ Characteristic } & \multicolumn{2}{|c|}{ WT } & \multicolumn{2}{|c|}{ IL-10 KO } \\
\hline & Male & Female & Male & Female \\
\hline Mean no. of OTU & $1,539.1^{\mathrm{a}}$ & $1,022.1^{\mathrm{a}}$ & 967.9 & $1,031.0$ \\
\hline Good's library coverage (\%) & 97.1 & 96.6 & 97.2 & 97.2 \\
\hline \multicolumn{5}{|l|}{ Alpha diversity } \\
\hline $\mathrm{ACE}$ & $2,139.2^{\mathrm{a}}$ & $4,129.2^{\mathrm{a}}$ & $2,368.7$ & $2,360.9$ \\
\hline Chaol & $1,851.4$ & $2,324.5$ & $1,725.2$ & $1,726.7$ \\
\hline Jackknife & $2,090.9$ & $2,935.6$ & $2,057.1$ & $2,023.2$ \\
\hline Shannon & $4.5391^{\mathrm{ab}}$ & $2.1775^{\mathrm{ac}}$ & $3.0426^{\mathrm{b}}$ & $3.2930^{\circ}$ \\
\hline Simpson & $0.0448^{\mathrm{ab}}$ & $0.4730^{\mathrm{ac}}$ & $0.1918^{\mathrm{b}}$ & $0.1221^{\mathrm{c}}$ \\
\hline Phylogenic diversity & $639.1^{\mathrm{b}}$ & 570.3 & $361.0^{\mathrm{b}}$ & 329.4 \\
\hline
\end{tabular}

OTU, operational taxonomic unit. ${ }^{\mathrm{a}} P<0.013$ for male wild type (WT) group vs. female WT group; ${ }^{\mathrm{b}} P<0.013$ for male WT group vs. male interleukin-10 (IL-10) knockout (KO) group; ${ }^{\mathrm{C}} P<0.013$ for female WT group vs. female IL-10 KO group; All after Holm-Bonferroni correction. 

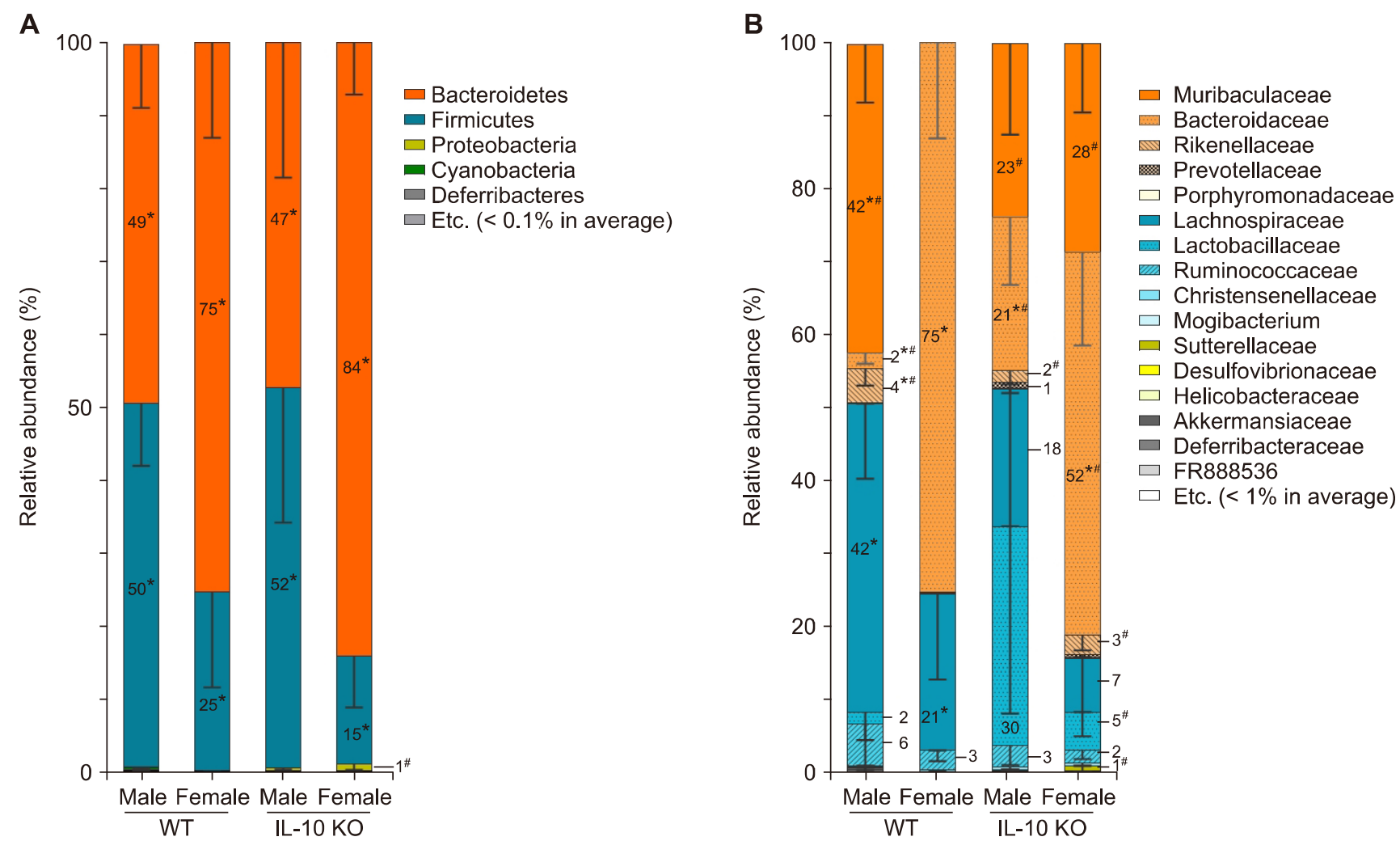

Figure 2. Gut microbiota composition at (A) phylum level and (B) family level in male and female mice of wild type (WT) groups and interleukin 10 (IL-10) knockout (KO) groups. ${ }^{*} P<0.013$ for male vs. female either in WT groups or IL-10 KO groups, ${ }^{\#} P<0.013$ for WT vs. IL-10 KO either in male or female groups after Holm-Bonferroni correction.

At family level, family Muribaculaceae was abundant in male WT mice while family Bacteroidaceae was prominent in female WT mice (Fig. 2B, 3E and 3F). Family Lachnospiraceae was dominant among other families of phylum Firmicutes in both male and female WT mice (Fig. 2B, 3H). The relative abundance of families Muriaculaceae and Rikenellaceae were also higher in female IL-10 KO mice compared to that in female WT mice (Fig. 3F and 3G). In contrast, family Bacteroidaceae was a lot more abundant in female WT mice than that in female IL-10 KO mice (Fig. 3E).

Results of LEfSe analysis also showed that genera Lactobacillus, PAC001068, KE159600, and Bacteroides significantly changed regarding to sex (Fig. 4A).

\section{Effects of interleukin 10 knockout on relative abundance of gut microbiota}

Phylum Proteobacteria was rarely detected in WT groups (Fig. 3D). Interestingly, it significantly increased in female IL-10 KO mice (Fig. 3D). Family Bacteroidaceae increased in male IL-10 KO mice compared to that in male WT mice while female groups showed the opposite tendency (Fig. 3E). Families Muriaculaceae and Rikenellaceae in male IL-10 KO mice showed lower relative abundance than those in male WT mice. However, these families increased in female IL-10 KO mice compared to those in female WT mice (Fig. 3F and 3G). In addition to the above-mentioned taxa, some bacterial groups with minor proportions also changed significantly according to IL-10 KO (Fig. 4B and 4C). Specifically, genus Alistipes decreased significantly in male IL-10 KO mice compared to male WT mice and genus PAC001043 decreased noticeably in female IL-10 KO mice comparted to its WT counterpart.

\section{Alterations of several specific fecal microbiota species in interleukin 10 knockout groups}

It was notable that family Lactobacillaceae had a high proportion in gut microbiota of male IL-10 KO mice (Fig. 2B). Among various species compromising family Lactobacillaceae, species Lactobacillus murinus had a dominant relative abundance in male IL-10 KO mice (Fig. 5A). Several specific species which are Bacteroides acidifaciens, Helicobacter hepaticus, and Parasutterella spp. prominently increased in IL-10 KO groups compared to WT groups (Fig. 5B-5D). Species B. acidifaciens scarcely presented in 

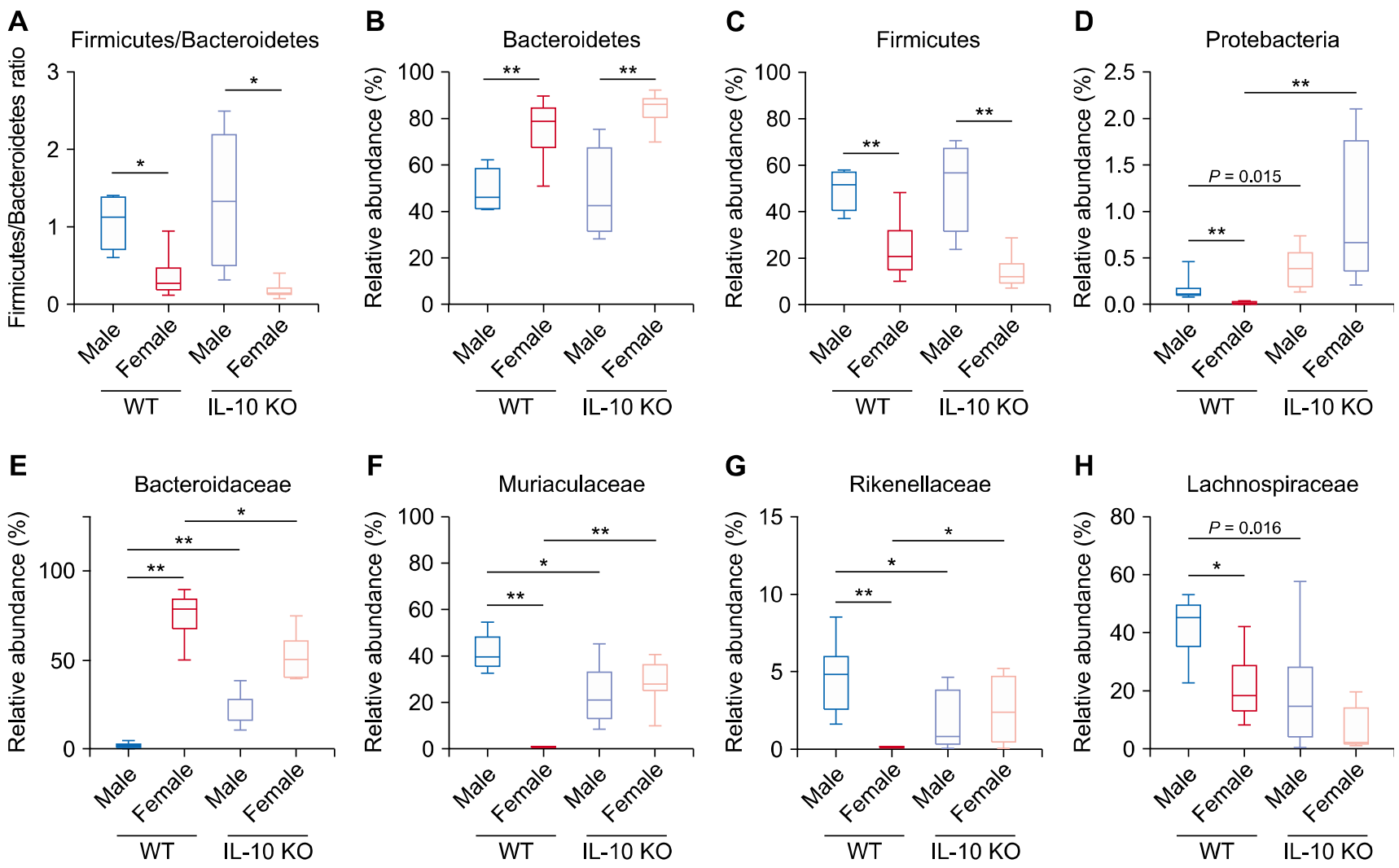

Figure 3. Relative abundance of specific taxa. Firmicutes to Bacteroides ratio (A) and relative abundance in major phylum (B-D) and family taxa (E-H). WT, wild type; IL-10, interleulin 10; KO, knockout. Kruskal-Wallis $P<0.05$ for (A-H). $* P<0.013, * * P<0.0025$ after HolmBonferroni correction.

WT groups, although it accounts for an average of $10 \%$ of gut microbiota in female IL-10 KO mice. Species $H$. hepaticuswas also rarely found in WT groups. However, its presence was significantly increased in both male and female IL-10 KO mice. The proportion of Parasutterella spp. was increased in female IL-10 KO mice compared to that in female WT mice.

\section{Higher relative abundance of beta-glucuronidase $(k 01195)$ in female mice}

According to PICRUSt, relative abundance of beta-glucuronidase (K01195) was higher in female groups than in male groups. Between female groups, female WT mice showed significantly increased relative abundance of beta-glucuronidase compared to female IL-10 KO mice.

\section{DISCUSSION}

Numerous diversity indices have been developed to estimate and describe characteristics of microbial communities. However, since each index was weighted on different aspects of diversity, diversity indices did not always show corresponding results [36]. In our study, while ACE indicating species richness was higher in female WT mice than that in male WT mice, Shannon and Simpson's indices taking account of both species richness and evenness indicated higher diversity in male WT mice compared to female WT mice. The low diversity in female WT mice might be due to a single species, Bacteroides vulgatus, which unexpectedly had an extremely high value (Fig. 5E). On the other hand, our previous study using F344 rat as an animal model did not show significant sex difference in microbial diversity [19]. This disparity might be due to differences in animal models, housing conditions, maternity, and contamination. Overall, these results demonstrate that we should be cautious when estimating microbial communities through diversity indices since each value cannot represent all communities completely. Further investigation with more controlled conditions is needed to thoroughly interpret effects of sex on microbial diversity.

IL-10 is a well-known anti-inflammatory cytokine which inhibits effects of helper $\mathrm{T}$ cells, natural killer cells, and macrophages [37]. Since IL-10 is accepted as a key repressor of 
A

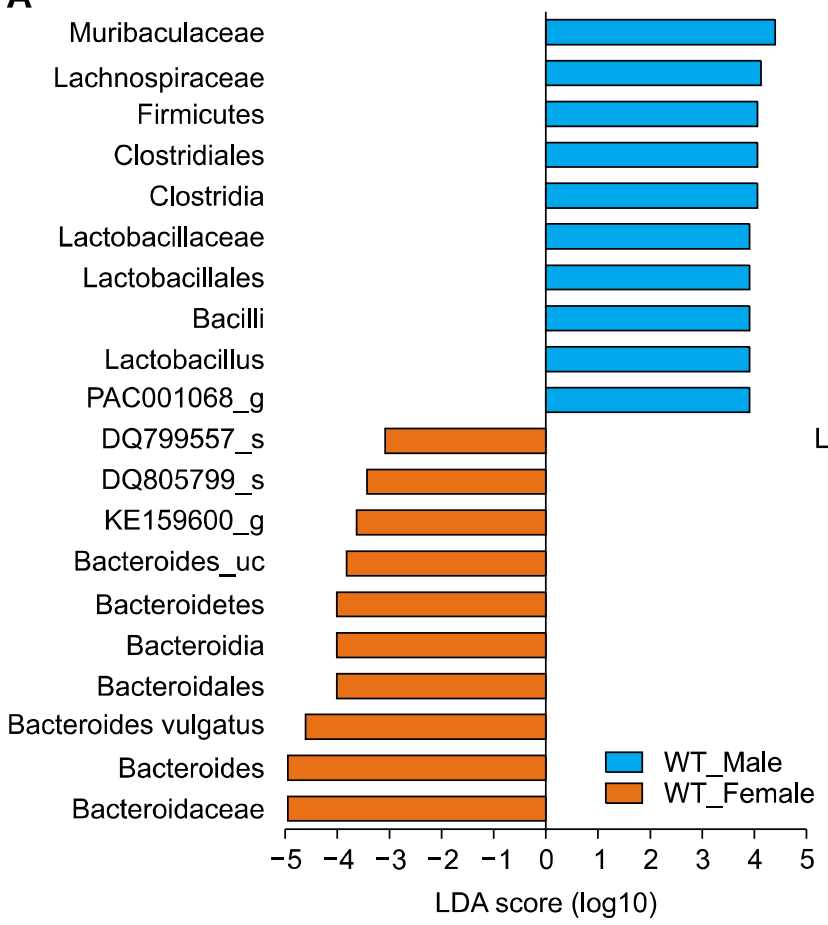

C

Bacteroidaceae

Bacteroides

Bacteroides vulgatus

Firmicutes

Lachnospiraceae_uc

PAC001232 s

PAC001091_g

KE159628_g

PAC000664_g

PAC001043_g

Lactobacillales

Bacilli

Lactobacillaceae

Bacteroidetes

Bacteroidia

Bacteroidales

Clostridia

Clostridiales

Lachnospiraceae

Muribaculaceae

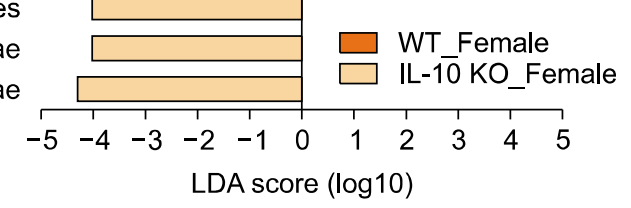

B

$$
\begin{array}{r}
\text { Muribaculaceae } \\
\text { Lachnospiraceae } \\
\text { Clostridiales } \\
\text { Clostridia } \\
\text { Bacteroidales } \\
\text { Bacteroidia } \\
\text { Bacteroidetes } \\
\text { PAC001060_s } \\
\text { Rikenellaceae } \\
\text { Alistipes }
\end{array}
$$

Lactobacillus murinus group

PAC001068_g

Lactobacillus

Lactobacillales

Bacilli

Lactobacillaceae

Firmicutes

Bacteroides vulgatus

Bacteroides

Bacteroidaceae

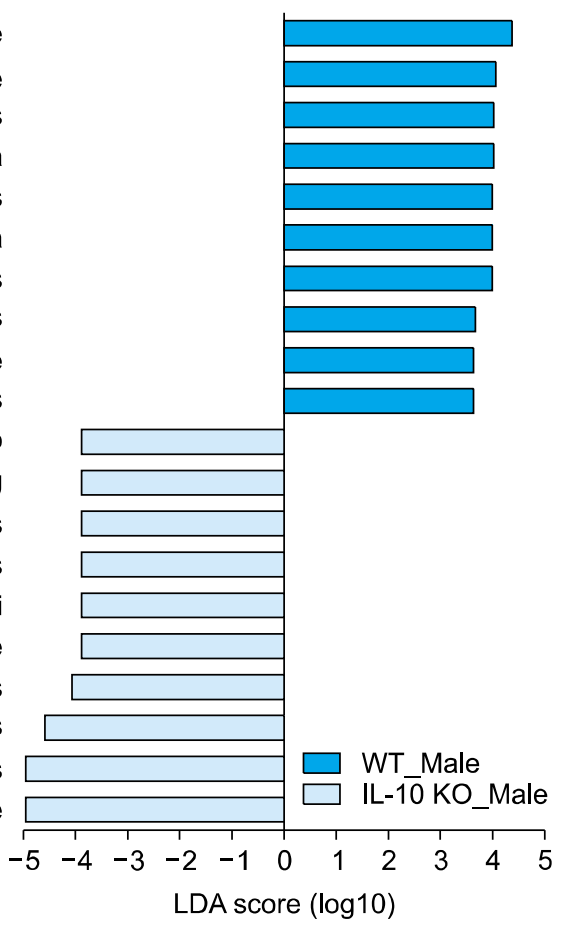

LDA score $(\log 10)$
Figure 4. Linear discriminant analysis (LDA) effect size analyses. (A) Male wild type (WT) mice and female WT mice, (B) male WT mice and male interleulin 10 (IL-10) knockout (KO) mice, and (C) female WT mice and female IL-10 KO mice. Only top ten and bottom ten taxa were presented. colitis, IL-10 KO mice model has been widely used as an IBD animal model [38]. IL-10 KO mice generally produce spontaneous colitis in conventional housing conditions [39-42]. IL-10 KO mice at 8-week-old showed significantly increased inflammatory markers, such as IL-1 $\beta$, IL-17, and myeloperoxidase and histologic score than that in WT mice (data not shown). IL-10 KO mice have unbalanced immune response in intestine which might lead to dysbiosis. However, in the present study, most diversity indices 
A

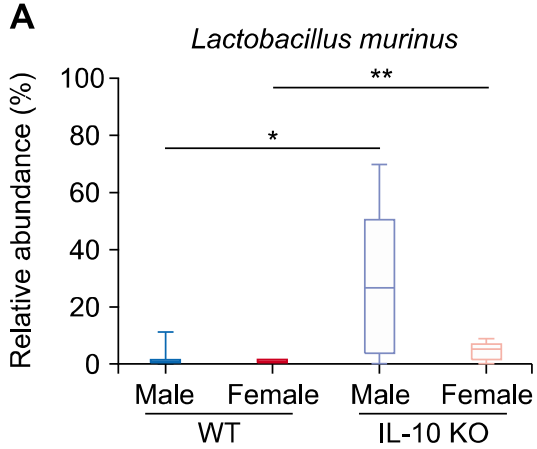

D

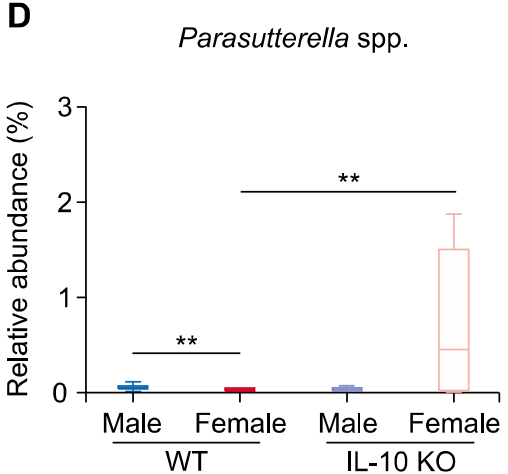

B

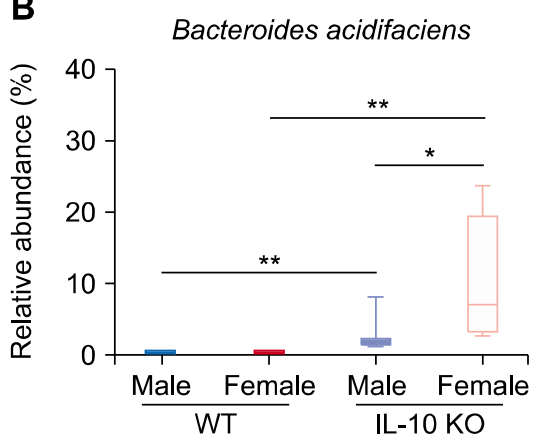

E

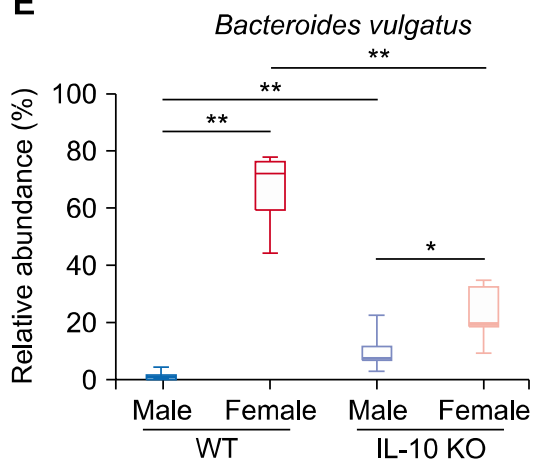

C

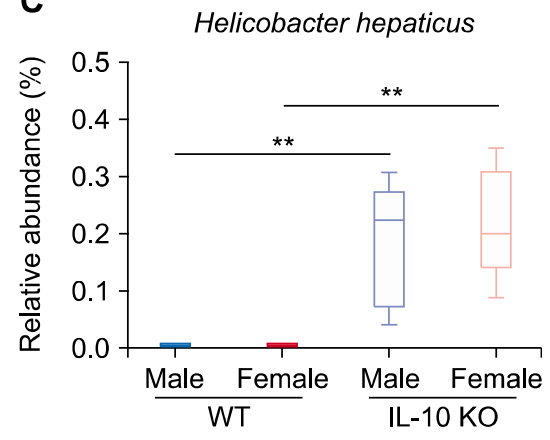

$\mathbf{F}$

$\beta$-glucuronidase [K01195]

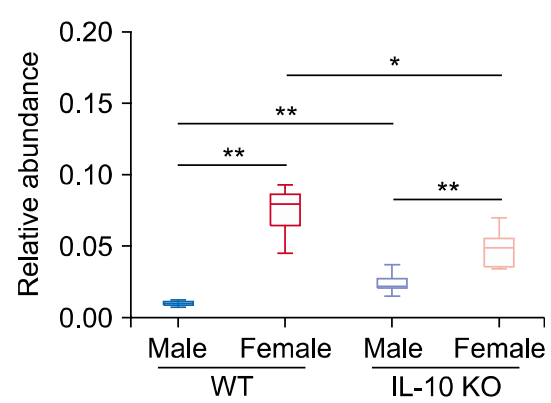

Figure 5. (A-D) Representative species increased in interleulin 10 (IL-10) knockout (KO) mice. (E) Bacteroides vulgatus increased in female mice. (F) Relative abundance of the Kyoto Encyclopedia of Genes and Genomes gene "beta-glucuronidaes" which is predicted by phylogenetic investigation of communities by reconstruction of unobserved states. WT, wild type. Kruskal-Wallis $P<0.05$ for (A-F). $* P<0.013$, ** $F$ $<0.0025$ after Holm-Bonferroni correction.

did not indicate significantly reduced diversity in IL-10 KO groups compared to WT groups (Table 1). In another study, IL-10 KO mice did not show any significant differences in Shannon or Simpson's index compared to WT mice either [43]. On the other hand, beta diversity analyses of PCOA and UPGMA tree divided WT groups and IL-10 KO groups into distinct groups (Fig. 1E and 1F). Difference between male IL-10 KO mice and female IL-10 Ko mice was less significant than that between male WT group and female WT group, suggesting that effect of IL-10 KO might have overwhelmed sex differences or that the interaction between sex hormones, such as estrogen and IL-10 might have been disrupted.

$\mathrm{F} / \mathrm{B}$ ratio is a representative index comparing different microbial communities [44]. However, reports regarding F/B ratios are varied among investigations. Many studies support the idea that a low $\mathrm{F} / \mathrm{B}$ ratio is related to a healthy condition $[44,45]$. However, IBD patients have decreased relative abundance of Firmicutes which usually indicates low $\mathrm{F} / \mathrm{B}$ ratio in several published reports [46-48]. Our results did not show any significant difference in $\mathrm{F} / \mathrm{B}$ ratio between WT mice and IL-10 KO mice. Instead, there were prominent differences of relative abundance and diversity of gut microbiota between male and female mice in both WT and IL-10 KO groups. This might be the result of unexpected high abundance of one species, $B$. vulgatus, as previously mentioned.

Phylum Proteobacteria increased in female IL-10 KO mice (Fig. 3D). It has been previously reported that phylum Proteobacteria is increased in IBD patients and that its invasive properties might destroy host defense mechanisms and provoke pro-inflammatory changes [49]. Although phylum Proteobacteria has a relatively small portion compared to phyla Firmicutes and Bacteroidetes, it might do role as a trigger of IBD initiation. In this context, changes in proportion of certain species, such as $B$. acidifaciens, $H$. hepaticus, and Parasutterella spp. can produce significant start points toward inflammatory cascades and eventually lead to the development of IBD (Fig. 5B-5D). However, we should always consider whether a change in gut microbiota is a cause or a result.

Beta-glucuronidase has been suggested as a key enzyme in estrobolome [22]. That is, beta-glucuronidase secreted by gut microbiota usually deconjugates metabolic materials in gut so that they are not excreted but reabsorbed. In case of estrogen, estriol-16-glucuronide passing through gut is deconjugated by bacterial beta-glucuronidase [22]. It then acts on various target 
organs such as intestine where estrogen is well known to repress inflammation [50]. The relative abundance of beta-glucuronidase predicted by PICRUSt was decreased in female IL-10 KO mice compared to that in female WT mice (Fig. 5F). These results collectively suggest that reduction of bacterial beta-glucuronidase caused by dysbiosis might lead to decrease of effective estrogen. In conclusion, our results suggest that microbiota-host interactions might differ between sexes during the development of IBD.

\section{ACKNOWLEDGMENTS}

This work was supported by a grant (NRF-2016R1A2B4013133) of the National Research Foundation (NRF) of Korea funded by the Ministry of Science, ICT and Future Planning.

\section{CONFLICTS OF INTEREST}

No potential conflicts of interest were disclosed.

\section{REFERENCES}

1. Podolsky DK. Inflammatory bowel disease. N Engl J Med 2002; 347:417-29.

2. Duerr RH, Taylor KD, Brant SR, Rioux JD, Silverberg MS, Daly MJ, et al. A genome-wide association study identifies IL23R as an inflammatory bowel disease gene. Science 2006;314:1461-3.

3. Oehlers SH, Flores MV, Hall CJ, Swift S, Crosier KE, Crosier PS. The inflammatory bowel disease (IBD) susceptibility genes NOD1 and NOD2 have conserved anti-bacterial roles in zebrafish. Dis Model Mech 2011:4:832-41.

4. Xavier RJ, Podolsky DK. Unravelling the pathogenesis of inflammatory bowel disease. Nature 2007:448:427-34.

5. Manichanh C, Borruel N, Casellas F, Guarner F. The gut microbiota in IBD. Nat Rev Gastroenterol Hepatol 2012;9:599-608.

6. Abraham C, Medzhitov R. Interactions between the host innate immune system and microbes in inflammatory bowel disease. Gastroenterology 2011;140:1729-37.

7. Sellon RK, Tonkonogy S, Schultz M, Dieleman LA, Grenther W, Balish E, et al. Resident enteric bacteria are necessary for development of spontaneous colitis and immune system activation in interleukin-10-deficient mice. Infect Immun 1998;66:5224-31.

8. Khan KJ, Ullman TA, Ford AC, Abreu MT, Abadir A, Marshall JK, et al. Antibiotic therapy in inflammatory bowel disease: a systematic review and meta-analysis. Am J Gastroenterol 2011;106: 661-73.

9. Cui B, Li P, Xu L, Peng Z, Xiang J, He Z, et al. Step-up fecal microbiota transplantation (FMT) strategy. Gut Microbes 2016;7:323-8.

10. Ott SJ, Musfeldt M, Wenderoth DF, Hampe J, Brant O, Fölsch UR, et al. Reduction in diversity of the colonic mucosa associated bacterial microflora in patients with active inflammatory bowel disease. Gut 2004:53:685-93.

11. Fujimoto T, Imaeda $\mathrm{H}$, Takahashi K, Kasumi E, Bamba S, Fujiyama $\mathrm{Y}$, et al. Decreased abundance of Faecalibacterium prausnitzii in the gut microbiota of Crohn's disease. J Gastroenterol Hepatol 2013;28:613-9.

12. Nishino K, Nishida A, Inoue R, Kawada Y, Ohno M, Sakai S, et al. Analysis of endoscopic brush samples identified mucosa-associated dysbiosis in inflammatory bowel disease. J Gastroenterol 2018:53:95-106.

13. Takahashi K, Nishida A, Fujimoto T, Fujii M, Shioya M, Imaeda H, et al. Reduced abundance of butyrate-producing bacteria species in the fecal microbial community in Crohn's disease. Digestion 2016;93:59-65.

14. Costello EK, Lauber CL, Hamady M, Fierer N, Gordon JI, Knight R. Bacterial community variation in human body habitats across space and time. Science 2009;326:1694-7.

15. Cardona S, Eck A, Cassellas M, Gallart M, Alastrue C, Dore J, et al. Storage conditions of intestinal microbiota matter in metagenomic analysis. BMC Microbiol 2012;12:158.

16. Ni J, Wu GD, Albenberg L, Tomov VT. Gut microbiota and IBD: causation or correlation? Nat Rev Gastroenterol Hepatol 2017;14: 573-84.

17. Plottel CS, Blaser MJ. Microbiome and malignancy. Cell Host Microbe 2011;10:324-35.

18. Blander JM, Longman RS, Iliev ID, Sonnenberg GF, Artis D. Regulation of inflammation by microbiota interactions with the host. Nat Immunol 2017;18:851-60.

19. Lee SM, Kim N, Yoon H, Nam RH, Lee DH. Microbial changes and host response in $\mathrm{F} 344$ rat colon depending on sex and age following a high-fat diet. Front Microbiol 2018;9:2236.

20. Macpherson AJ, Harris NL. Interactions between commensal intestinal bacteria and the immune system. Nat Rev Immunol 2004:4:478-85.

21. Round JL, Mazmanian SK. The gut microbiota shapes intestinal immune responses during health and disease. Nat Rev Immunol 2009:9:313-23.

22. Baker JM, Al-Nakkash L, Herbst-Kralovetz MM. Estrogen-gut microbiome axis: physiological and clinical implications. Maturitas 2017; 103:45-53.

23. Shah SC, Khalili H, Gower-Rousseau C, Olen O, Benchimol EI, Lynge $\mathrm{E}$, et al. Sex-based differences in incidence of inflammatory bowel diseases-pooled analysis of population-based studies from Western countries. Gastroenterology 2018;155:1079-89.e3.

24. Shah SC, Khalili H, Chen CY, Ahn HS, Ng SC, Burisch J, et al. Sex-based differences in the incidence of inflammatory bowel diseases-pooled analysis of population-based studies from the Asia-Pacific region. Aliment Pharmacol Ther 2019;49:904-911.

25. Bolger AM, Lohse M, Usadel B. Trimmomatic: a flexible trimmer for Illumina sequence data. Bioinformatics 2014;30:2114-20.

26. Masella AP, Bartram AK, Truszkowski JM, Brown DG, Neufeld JD. PANDAseq: paired-end assembler for illumina sequences. BMC Bioinformatics 2012;13:31.

27. Eddy SR. Accelerated profile HMM searches. PLoS Comput Biol 2011;7:e1002195.

28. Lee B, Moon T, Yoon S, Weissman T. DUDE-Seq: fast, flexible, and robust denoising for targeted amplicon sequencing. PLoS One 2017;12:e0181463.

29. Edgar RC. Search and clustering orders of magnitude faster than BLAST. Bioinformatics 2010;26:2460-1.

30. Myers EW, Miller W. Optimal alignments in linear space. Comput Appl Biosci 1988:4:11-7.

31. Edgar RC, Haas BJ, Clemente JC, Quince C, Knight R. UCHIME im- 
proves sensitivity and speed of chimera detection. Bioinformatics 2011:27:2194-200.

32. Fu L, Niu B, Zhu Z, Wu S, Li W. CD-HIT: accelerated for clustering the next-generation sequencing data. Bioinformatics 2012;28:3150-2.

33. Yoon SH, Ha SM, Kwon S, Lim J, Kim Y, Seo H, et al. Introducing EzBioCloud: a taxonomically united database of 16S rRNA gene sequences and whole-genome assemblies. Int J Syst Evol Microbiol 2017:67:1613-7.

34. Segata N, Izard J, Waldron L, Gevers D, Miropolsky L, Garrett WS, et al. Metagenomic biomarker discovery and explanation. Genome Biol 2011;12:R60

35. Langille MG, Zaneveld J, Caporaso JG, McDonald D, Knights D, Reyes JA, et al. Predictive functional profiling of microbial communities using 16S rRNA marker gene sequences. Nat Biotechnol 2013;31:814-21.

36. Kim BR, Shin J, Guevarra R, Lee JH, Kim DW, Seol KH, et al. Deciphering diversity indices for a better understanding of microbial communities. J Microbiol Biotechnol 2017;27:2089-93.

37. Chaudhry A, Samstein RM, Treuting P, Liang Y, Pils MC, Heinrich $\mathrm{JM}$, et al. Interleukin-10 signaling in regulatory $\mathrm{T}$ cells is required for suppression of Th17 cell-mediated inflammation. Immunity 2011;34:566-78.

38. Mizoguchi A, Mizoguchi E. Animal models of IBD: linkage to human disease. Curr Opin Pharmacol 2010;10:578-87.

39. Kühn R, Löhler J, Rennick D, Rajewsky K, Müller W. Interleukin10-deficient mice develop chronic enterocolitis. Cell 1993;75: 263-74.

40. Berg DJ, Davidson N, Kühn R, Müller W, Menon S, Holland G, et al. Enterocolitis and colon cancer in interleukin-10-deficient mice are associated with aberrant cytokine production and CD4(+) TH1-like responses. J Clin Invest 1996;98:1010-20.

41. Singh UP, Singh S, Singh R, Cong Y, Taub DD, Lillard JW Jr. CXCL10-producing mucosal CD4+ T cells, NK cells, and NKT cells are associated with chronic colitis in IL-10(-/-) mice, which can be abrogated by anti-CXCL10 antibody inhibition. J Interferon Cytokine Res 2008:28:31-43.

42. Keubler LM, Buettner M, Häger C, Bleich A. A multihit model: colitis lessons from the interleukin-10-deficient mouse. Inflamm Bowel Dis 2015;21:1967-75.

43. Zhang T, Ding C, Zhao M, Dai X, Yang J, Li Y, et al. Sodium butyrate reduces colitogenic immunoglobulin a-coated bacteria and modifies the composition of microbiota in IL-10 deficient mice. Nutrients 2016;8:E728.

44. Mariat D, Firmesse O, Levenez F, Guimarăes V, Sokol H, Doré J, et al. The Firmicutes/Bacteroidetes ratio of the human microbiota changes with age. BMC Microbiol 2009;9:123.

45. Turnbaugh PJ, Ley RE, Mahowald MA, Magrini V, Mardis ER, Gordon JI. An obesity-associated gut microbiome with increased capacity for energy harvest. Nature 2006;444:1027-31.

46. Rehman A, Lepage P, Nolte A, Hellmig S, Schreiber S, Ott SJ. Transcriptional activity of the dominant gut mucosal microbiota in chronic inflammatory bowel disease patients. J Med Microbiol 2010;59:1114-22.

47. Frank DN, St Amand AL, Feldman RA, Boedeker EC, Harpaz N, Pace NR. Molecular-phylogenetic characterization of microbial community imbalances in human inflammatory bowel diseases. Proc Natl Acad Sci U S A 2007; 104:13780-5.

48. Gophna U, Sommerfeld K, Gophna S, Doolittle WF, Veldhuyzen van Zanten SJ. Differences between tissue-associated intestinal microfloras of patients with Crohn's disease and ulcerative colitis. J Clin Microbiol 2006;44:4136-41.

49. Mukhopadhya I, Hansen R, El-Omar EM, Hold GL. IBD-what role do Proteobacteria play? Nat Rev Gastroenterol Hepatol 2012;9: 219-30.

50. Son HJ, Sohn SH, Kim N, Lee HN, Lee SM, Nam RH, et al. Effect of estradiol in an azoxymethane/dextran sulfate sodium-treated mouse model of colorectal cancer: implication for sex difference in colorectal cancer development. Cancer Res Treat 2019;51:632-48. 